\title{
Ocorrência de Atelocynus microtis (Sclater, 1882) na Floresta Nacional do Jamari, estado de Rondônia
}

\author{
Allyson Diaz Koester ${ }^{1,3}$, Carlos Renato de Azevedo ${ }^{2}$, \\ Alexandre Vogliotti ${ }^{1,3}$ \& José Maurício Barbanti Duarte ${ }^{3,4}$ \\ ${ }^{1}$ Programa de Pós-graduação em Ecologia Aplicada, \\ Escola Superior de Agricultura “Luiz de Queiroz”- USP, \\ Av. Pádua Dias, 11, CP 9, CEP 13418-900, Piracicaba, SP, Brasil \\ ${ }^{2}$ Floresta Nacional do Jamari, ICMBio /MMA, \\ RO 452, km 7.5, Zona Rural, CEP 78937-000, Itapuã do Oeste, RO, Brasil \\ ${ }^{3}$ Núcleo de Pesquisa e Conservação de Cervídeos, \\ Faculdade de Ciências Agrárias e Veterinárias - UNESP, \\ Via de acesso Prof. Paulo Donato Castellane, s/n, CEP 14884-900, Jaboticabal, SP, Brasil \\ ${ }^{4}$ Autor para correspondência: José Maurício Barbanti Duarte, email: barbanti@fcav.unesp.br
}

KOESTER, A.D., AZEVEDO, C.R., VOGLIOTTI, A., \& DUARTE, J.M.B. Occurrence of Atelocynus microtis (Sclater, 1882) in the Jamari National Forest, Rondonia state. Biota Neotrop., 8(4): http://www.biotaneotropica. org.br/v8n4/en/abstract?short-communication+bn03108042008

\begin{abstract}
The Short-eared dog Atelocynus microtis is one of the most rare species of South America Canids. It is rarely reported in inventories or faunal surveys and the absence of captive animals also suggest a restrict distribution and low densities. Between August $16^{\text {th }} 2006$ and October 12 $2^{\text {th }}$ 2006, a photographic monitoring using camera traps (infrared-triggered), was carried out at the Jamari National Forest. This is a sustainable use conservation unity and has a management plan with its defined environmental zones. The Atelocynus microtis photos were captured in the Central-west and North-west areas of the Jamari National Forest, areas constituted by Dense Evergreen Forest with Open Evergreen Forest spots. The areas where the images of A. microtis were captured are in forest management zone and in mining zone, which still not exploited. Based on these facts, we suggest the management plan of this Conservation Unity took this finding into consideration and altered it, so this species could be better studied in the region, before its exploitation. As there are hardly any studies related to this species, this note becomes important, contributing to data about the A. microtis ecology.

Keywords: Atelocynus microtis (short-eared dog), photographic monitoring, Jamari National Forest.
\end{abstract}

KOESTER, A.D., AZEVEDO, C.R., VOGLIOTTI, A., \& DUARTE, J.M.B. Ocorrência de Atelocynus microtis (Sclater, 1882) na Floresta Nacional do Jamari, estado de Rondônia. Biota Neotrop., 8(4): http://www. biotaneotropica.org.br/v8n4/pt/abstract?short-communication+bn0310808042008

Resumo: O cachorro-de-orelhas-curtas Atelocynus microtis é uma das espécies mais raras de Canídeos Sulamericanos. Os relatos são muito raros e a inexistência de animais cativos também sugere distribuição limitada e densidades baixas. No período de 16 de agosto a 12 de outubro de 2006, foi realizado um monitoramento fotográfico na Floresta Nacional do Jamari, que é uma Unidade de Conservação de Uso Sustentável e possui plano de manejo com seu zoneamento ambiental definido. Os registros fotográficos de Atelocynus microtis foram obtidos nas regiões centro-oeste e noroeste da unidade, áreas formadas por Floresta Ombrófila Densa, com pontos de Floresta Ombrófila Aberta. As áreas onde foram capturadas as imagens fotográficas de Atelocynus microtis, estão em Zona de Manejo Florestal e em Zona de Mineração, por enquanto sem exploração. A partir destes fatos, a sugestão seria que o zoneamento ambiental desta Unidade de Conservação levasse em consideração este achado e fosse alterado para que a espécie fosse mais bem estudada na região antes da exploração das áreas. Como estudos relacionados a esta espécie são praticamente inexistentes, esta nota se torna importante, contribuindo com dados sobre a ecologia do A. microtis.

Palavras-chave: Atelocynus microtis (cachorro-do-mato-de-orelhas-curtas), monitoramento fotográfico, Floresta Nacional do Jamari. 


\section{Introdução}

Pertencente a Ordem Carnívora, o cachorro-de-orelhas-curtas, Atelocynus microtis, Sclater (1882), é a única espécie do seu gênero (Berta, 1986). Sua área de ocorrência se estende desde a Colombia, Bolívia, Equador, Peru e Brasil, até o norte do MatoGrosso (Nowak \& Paradiso 1983, Berta 1986, Emmons \& Feer 1997, Eisenberg \& Redford 1999, Cheida et al. 2003). Berta (1986) cita a ocorrência da espécie na Venezuela, mas de acordo com Leite-Pitman \& Willians (2004) essa informação não foi comprovada. O A. microtis está disperso, por grande parte da bacia amazônica, chegando à bacia do rio Paraguai, através do rio Araguaia-Tocantins (Emmons \& Feer 1997, Paiva 1999), em regiões com até $1.000 \mathrm{~m}$ de altitude em relação ao nível do mar (Berta 1986, Peres 1991, Cheida et al. 2003, Leite-Pitman \& Willians 2004).

Segundo Sillero-Zubiri \& Hoffmann (2004) a espécie tem sido registrada em uma ampla variedade de habitats das terras baixas, incluindo floresta de terra firme, floresta inundada, bambuzal e mata ciliar ao longo dos rios. Os autores ainda relatam que em Cocha Cashu, visualizações e rastros da espécie estão principalmente associados aos rios e as enseadas dos mesmos. No Estado de Rondônia, M. Messias (com. pes.) informou a visualização de um indivíduo em floresta das terras baixas beirando a savana, na Estação Ecológica de Samuel. Além desta, no ano de 2007, A. R. D’ Amico (com pes.) visualizou a espécie em floresta primária das terras baixas e com influência hídrica marcante na Reserva Biológica Jaru.

De acordo com Cabrera \& Yepes (1960), Peres (1991) e Eisenberg \& Redford (1999) a espécie possui dieta onívora e hábitos solitários, e foi apontada por estes autores como o menos gregário dos canídeos sul-americanos. Esta espécie ainda sofre ameaças por doenças transmitidas por animais domésticos e pela perda de habitat em decorrência da degradação das florestas (Cheida et al. 2003, Sillero-Zubiri \& Hoffmann 2004).

Estudos relacionados ao A. microtis são praticamente inexistentes (Peres 1991, Leite-Pitman \& Willians 2004). Peres (1991) cita que a espécie é raramente relatada em inventários de fauna, e sua ocorrência é freqüentemente classificada como hipotética ou a confirmar. Por essas razões, a espécie encontra-se categorizada como DD (Data Deficient) pela IUCN (Sillero-Zubiri \& Hoffmann 2004).

Frente à escassez de dados sobre a espécie, o presente relato pretende contribuir para o conhecimento da espécie, adicionando mais uma localidade com ocorrência confirmada à distribuição geográfica da espécie.

\section{Material e Métodos}

\section{1. Área de estudo}

A Floresta Nacional do Jamari (FLONA do Jamari) situada no município de Itapuã do Oeste, no estado de Rondônia, é uma Unidade de Conservação Federal de Uso Sustentável (Sistema Nacional de Unidades de Conservação/SNUC, 2000). Possui 220.000 ha e sua situação fundiária está regularizada. Localizada no norte do estado de

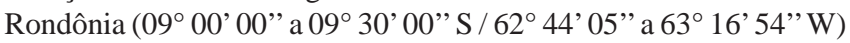
é banhada pelas bacias dos rios Jacundá, Jamari e Preto do Crespo. Esta Unidade resguarda amostras da flora características da Amazônia sul-ocidental, região submetida a altas taxas de desmatamento. Juntamente com a Floresta Nacional do Jacundá (220.644 ha), a Estação Ecológica de Samuel (72.000 ha) e o Imóvel Manoa (73.079 ha), a Floresta Nacional do Jamari faz parte de uma área contínua bastante significativa de Floresta Amazônica (Floresta Tropical) no estado de Rondônia, Brasil.

A área específica de estudo foram as regiões centro-oeste e noroeste da unidade. A formação vegetal que prevalece nestas áreas é a Floresta Ombrófila Densa/Aberta das terras baixas, com fascinações de Floresta de Baixio. Esta formação vegetal recobre áreas de 70 a 160 m de altitude (Plano de Manejo da Floresta Nacional do Jamari, 2005).

De acordo com a classificação de Koppen esta região possui clima do tipo Aw - Tropical chuvoso, com período seco bem definido durante o inverno. A média anual de precipitação pluvial varia de 2.200 e $2.600 \mathrm{~mm}$ por ano. A temperatura média anual fica entre 24 e $26^{\circ} \mathrm{C}$.

\section{Monitoramento fotográfico}

No período de 16 de agosto a 12 de outubro de 2006, foi realizado um monitoramento fotográfico na Floresta Nacional do Jamari/ Ibama, inicialmente com o objetivo de estudar espécies da Família Cervidae.

Para este estudo foram utilizadas 15 armadilhas fotográficas da marca Tigrinus ${ }^{\circledR}$ (modelo 4.0C, Timbó, SC, BRA.) e 05 da marca Cam Trak (South Inc., Watkinsville, GA, EUA.). Os pontos de instalação foram escolhidos dentre um conjunto de rotas previamente identificadas, em função dos indícios da presença de veados (pegadas, fezes, fruteiras reviradas). Todos os pontos foram georreferenciados com o auxílio de um GPS Garmin Plus III.

Os equipamentos foram instalados a uma altura de $40 \mathrm{~cm}$ do solo e programados para registrar o dia e o horário de cada fotografia, funcionando em período integral, com um intervalo mínimo de 20 segundos entre fotografias subseqüentes. A verificação e manutenção do equipamento ocorreram semanalmente.

\section{Resultados}

Apenas duas ocorrências de A. microtis, foram registradas após um esforço amostral de 21.709,4 horas de monitoramento. As fotos foram obtidas nas coordenadas $09^{\circ} 04^{\prime} 58.7^{\prime \prime} \mathrm{S} / 063^{\circ} 06^{\prime} 41.3^{\prime \prime} \mathrm{W}$ e $09^{\circ} 11$ ' $34.8^{\prime \prime} \mathrm{S} / 063^{\circ} 06^{\prime} 11.3^{\prime}$ "W, distantes $12 \mathrm{~km}$ uma da outra (Figura 1).

Os registros ocorreram em área de floresta de terras baixas com forte influência hídrica, sob exemplares de Parkia multijuga (paricá) e Bellucia grossularioides (goiaba-de-anta) localizadas em zonas de Manejo Florestal e de Mineração ainda não exploradas na FLONA. As imagens (Figura 2) foram capturadas em dias e horários diferentes, sendo a primeira imagem registrada no dia 13/09/2006 as 15:29 h e a segunda registrada dia 20/09/2006 as 06:49 h.

Outros mamíferos raros e ameaçados registrados nesse monitoramento fotográfico foram: Priodontes maximus, Myrmecophaga tridactyla, Panthera onca, Puma concolor e Leopardus pardalis.

\section{Discussão}

A Floresta Nacional do Jamari é uma Unidade de Conservação de Uso Sustentável (Sistema Nacional de Unidades de Conservação/ SNUC, 2000) e possui plano de manejo com seu zoneamento ambiental definido (Plano de Manejo da Floresta Nacional do Jamari, 2005). As áreas onde foram capturadas as imagens fotográficas de A. microtis, de acordo com o Plano de Manejo da Floresta Nacional do Jamari (2005), estão na Zona de Manejo Florestal (105.475 ha) e na Zona de Mineração (16.446 ha), por enquanto sem exploração.

De acordo com Primack \& Rodrigues (2001), a partir do momento em que uma área de proteção é legalmente estabelecida, ela deve ser eficazmente manejada se quisermos que a diversidade biológica seja mantida. Segundo os autores o ponto crucial é que as unidades de conservação, às vezes, precisam ser ativamente manejadas para evitar sua deterioração. Porém, deixam claro que as decisões sobre o manejo de uma unidade de conservação podem ser tomadas mais 
eficazmente quando as informações são fornecidas por um programa de pesquisas.

O fato da Floresta Nacional do Jamari, bem como as demais Unidades de Conservação e o Imóvel ao seu redor serem áreas bastante irrigadas por igarapés, corroboram as informações de Berta (1986) e Sillero-Zubiri \& Hoffmann (2004), que sugerem que A. microtis

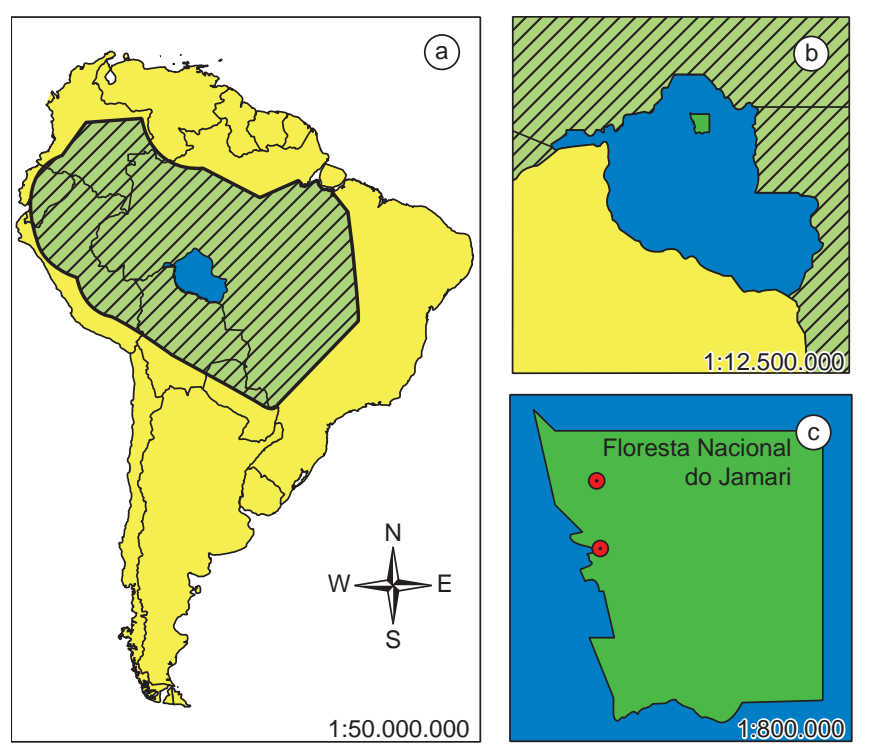

$\odot$ Registros do Atelocynus microtis na Floresta Nacional do Jamari
$\square$ Floresta Nacional do Jamari
$\square$ Rondônia
$\square$ América do Sul
$\square$ Distribuição do Atelocynus microtis na América do Sul

Figura 1. a) Distribuição do Atelocynus microtis na América do Sul (Berta, 1986), b) Localização da Floresta Nacional do Jamari no Estado de Rondônia, e c) Localização dos pontos onde Atelocynus microtis foi fotografado na Floresta Nacional do Jamari.

Figure 1. a) Distribution of Atelocynus microtis in South America (Berta, 1986), b) Position of Jamari National Forest on Rondonia State, and c) Sampling sites of Atelocynus microtis on the Jamari National Forest. está freqüentemente relacionado à ambientes aquáticos ou de florestas tropicais de grande umidade.

Nowak \& Paradiso (1983) acredita que A. microtis apresenta hábitos noturnos, enquanto Emmons \& Feer (1997) sugerem hábitos diurnos e crepusculares. Para Cheida et al. (2003) e Leite-Pitman \& Willians (2004), a espécie apresenta hábitos tanto diurnos como noturnos. Apesar do pequeno número de registros aqui apresentados, foi registrada apenas atividade diurna para a espécie.

As informações sobre a biologia básica dos organismos têm um papel essencial na conservação (Primack \& Rodrigues, 2001). Portanto, o desconhecimento sobre aspectos biológicos básicos de A. microtis pode comprometer o desenvolvimento de programas de conservação consistentes para a espécie. O presente trabalho contribui com essa lacuna de conhecimento, adicionando uma localidade importante à distribuição geográfica de $A$. microtis.

\section{Agradecimentos}

O trabalho de campo foi desenvolvido com o apoio financeiro da Fundação de Amparo a Pesquisa do Estado de São Paulo (FAPESP), CNPq e FUNEP. Em especial agradecemos ao Sr. Dejesus Aparecido Ramos, pelo apoio no campo sem o qual o trabalho se tornaria mais lento e difícil.

\section{Referências Bibliográficas}

BERTA, A. 1986. Atelocynus microtis. Mammal. Spec. 256:1-3.

CABRERA, A. \& YEPES, J. 1960. Mamíferos Sudamericanos. 2 ed. Editora Ediar, Buenos Aires.

CHEIDA, C.C., NAKARO, E.O., QUADROS, J., COSTA, R.F. \& ROCHA, F.M. 2003. Ordem Carnivora. In Mamíferos do Brasil. (N.R. Reis, A.L. Peracchi, W.A. Pedro \& I.P. Lima, eds.). Londrina, p. 242-275.

EISENBERG, J. \& REDFORD, K.H. 1999. Mammals of the Neotropics. The Central Neotropics: Ecuador, Peru, Bolivia, Brazil. v. 3. The University of Chicago Press, Chicago, p. 279-281.

EMMONS, L.H. \& FEER, F. 1997. Neotropical rainforest mammals: A field guide. 2 ed. The University of Chicago Press, Chicago.

LEITE-PITMAN, M.R.P. \& WILLIANS, R.S.R. 2004. The short-eared dog (Atelocynus microtis: Sclater 1883). In Canids: Foxes, Wolves, Jackals and Dogs. Status Survey and Conservation Action Plan. (C. Sillero-Zubiri, M. Hoffmann, D.W. MacDonald \& the IUCN/SSC Canid specialist Group, eds.). IUCN, Gland e Cambrigde, p. 26-31.
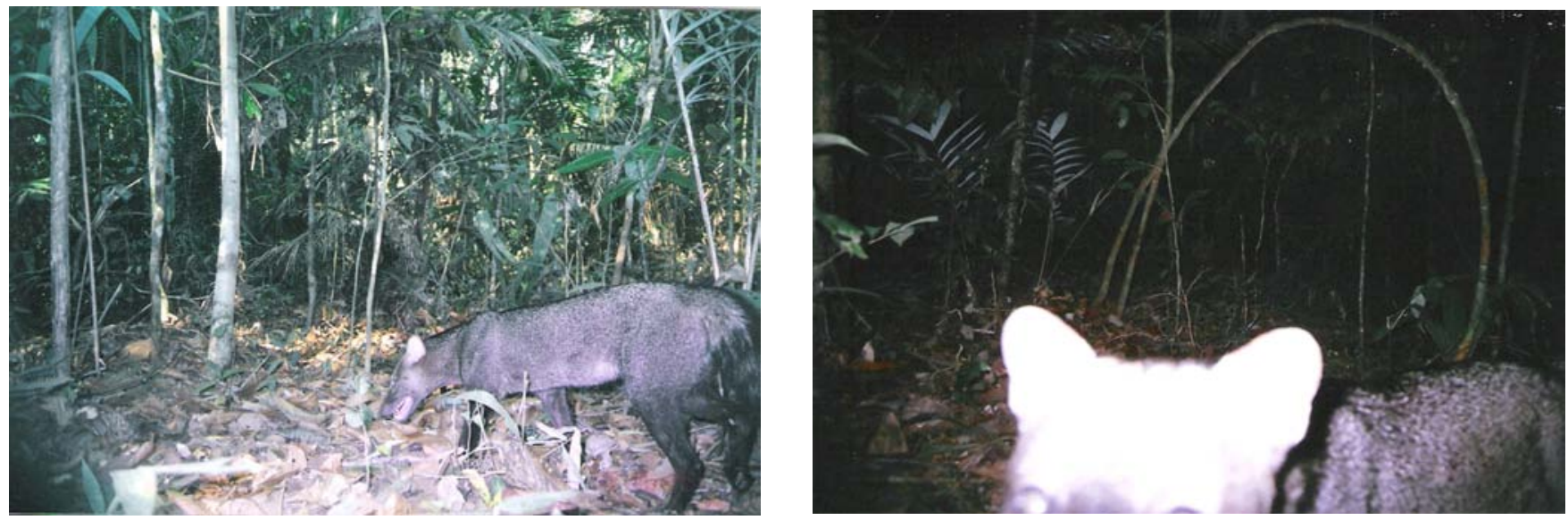

Figura 2. Registros fotográficos de Atelocynus microtis na Floresta Nacional do Jamari/RO.

Figure 2. Photographic records of Atelocynus microtis in Jamari National Forest/RO. 
NOWAK, R.M \& PARADISO, J.L. 1983. Walker's mammals of the World. 4 ed. The Johns Hopkins University Press, Baltimore.

PAIVA, M.P. 1999. Conservação da fauna brasileira. Editora Interciência, Rio de Janeiro.

PERES, C.A. 1991. Observations on hunting by small-eared (Atelocynus microtis) and bush dogs (Speothos venaticus) in central-western Amazonia. Mammalia. 55(4):635-639.

PLANO DE MANEJO DA FLORESTA NACIONAL DO JAMARI. 2005. Edições Ibama/MMA, Brasília.

PRIMACK, R.B. \& RODRIGUES, E. 2001. Biologia da Conservação. Editora Midiograf, Londrina.
SILLERO-ZUBIRI, C. \& HOFFMANN, M. 2004. Atelocynus microtis. In 2007 IUCN Red List of Threatened Species: http://www.iucnredlist.org. (último acesso em: 30/11/2007).

SISTEMA NACIONAL DE UNIDADES DE CONSERVAÇÃO/SNUC. 2000. Lei 9.985. Edições Ibama/MMA, Brasília.

Recebido em 06/04/08 Versão Reformulada recebida em 19/11/08

Publicado em 29/12/08 